\title{
The influence of an abdominal support for a dental stool in the distributions and electrical activity of the longissimus and the superior trapezius muscle in dentists
}

\author{
Custódio RAR ${ }^{\mathrm{a},{ }^{*}, \text { Brandão JGT }^{\mathrm{a}} \text { and Amorim JBO }}{ }^{\mathrm{b}}$ \\ ${ }^{a}$ Deparment of Mechanical, State University of São Paulo, Av. Dr. Ariberto Pereira da Cunha, 333 \\ Guaratinguetá-SP, Brazil \\ ${ }^{b}$ Deparment of Biosciences and Oral Diagnosis, State University of São Paulo, Av. Francisco José Longo, 777 \\ São José dos Campos-SP, Brazil
}

\begin{abstract}
This study evaluated the influence an abdominal support attached to a traditional stool, of those used by dentists, has on the body's distribution of the electrical activity of the superior trapezius and the longissimus thoracic muscles of dental students during the execution of a clinical procedure. The results showed no significant difference in the body's distribution in the seat and backrest, but did reveal there was a weight discharge of $3.1 \pm 1.9 \%$ of dentist's body weight in the abdominal support. The 9 o'clock position proved to be the best position to perform clinical procedures. It was also observed that the position was closer to the body's axis.
\end{abstract}

Keywords: electromyography; extensomety; dentistry

\section{Introduction}

The industrial studies concerning comfort in the seated position and chair design usually emphasize the inclination of the backrest. However, in Dentistry, it is impractical to work in an inclined position, for it cancels out the function of a back support when performing a clinical procedure [2]. The forward-right inclined position is identified as the most common position among dentists [3]. Remaining in this position and the length of time in it are considered aggravating factors for musculoskeletal pain and discomfort. It is also known that there is an increase of compression on the intervertebral discs and tension on the passive element of the spine when in positions which are far from the body's central axis [7]. Among these professionals, a high index of musculoskeletal discomfort is seen and found in the neck (87\%), lumbar spine $(63.3 \%)$, shoulders $(53 \%)$ and wrists $(36.7 \%)$ [4].

Providing support for the lumbar spine when the trunk is inclined forward to access the oral cavity becomes a challenging issue [1]. Therefore, when considering the effort in trunk flexion, this study aims to determine whether the use of an abdominal support interferes with the dentist's electromyographic activity in terms of the

\footnotetext{
${ }^{*}$ Corresponding author. Tel: 553591911469 E-mail: renatacustodio2005@yahoo.com.br
} 


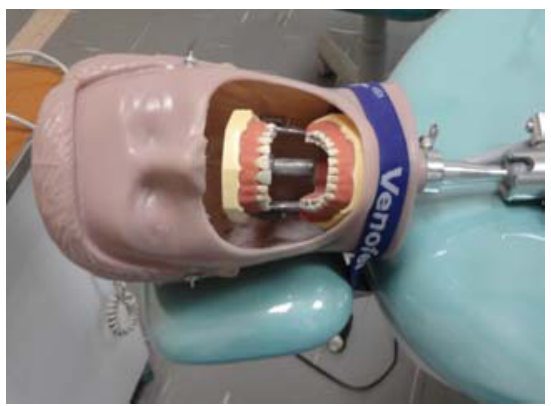

Figure 1: Dental dummy

superior trapezius and longissimus thoracic muscles, and the distribution of body weight on a dental stool in order to promote a position closer to the central axis while performing a restorative procedure on a dental dummy (Figure 1).

\section{Method}

The study was performed with 10 female dental students as respondents, aged $25 \pm 3.30$, with a body mass index (BMI) of $21.70 \pm 2.44 \mathrm{~kg} / \mathrm{m}^{2}$. Nine of the respondents are right-handed and one is left-handed. An industrial dental stool (Dabi Atlante, Ergorelax model) was instrumented with six load cells; one in the backrest $(\mathrm{Br})$, one in an abdominal support $(\mathrm{AbS})$, two in the front of the seat (FS) and two in back of the seat (BS) (Figure 2). The cells formed a complete Wheatstone bridge, according to the extensometer theory in each part cited.

In order to acquire the force signals applied to FS, $\mathrm{BS}, \mathrm{Br}$ and $\mathrm{AbS}$, a Spider 8 signal conditioning system was used, as well as the Catman 3.1 software (HBM/Darmstadt, Germany). The cells were calibrated and showed good linearity $\left(\mathrm{R}^{2}=0,9998\right.$ - FS, $\mathrm{R}^{2}=0.9997-\mathrm{BS}, \mathrm{R}^{2}=1-\mathrm{Br}$ and $\left.\mathrm{AbS}\right)$. Active surface electrodes of Silver Chloride $(\mathrm{AgCi})$ with a diameter of $1 \mathrm{~cm}$ were used to acquire the electromyographic signal (EMG) of the longissimus thoracic muscles at the first lumbar vertebra (L1) and the superior trapezius. The EMG device used was the EMG-810C (Brazil System) with six channels and by following the SENIAM (Surface Electromyography for the Non-Invasive Assessment of Muscles) protocol [5]. The volunteers signed an Informed Consent Form. This study was approved by the Ethics Committee of the State University of São Paulo/São José dos Campos.

\section{Results}

Direct vision was preferred by $90 \%$ of the respondents studied. The adopted working position according to the International Organization for Standardization (ISO) and the Federation Dentaire International (FDI) was the 9 o'clock position for $30 \%$ of dentists, 10 o'clock for $40 \%$ of these professionals and 11 o'clock for $30 \%$. The statistical analyses show an increase in electrical activity of the right trapezius muscles $(p<0.05)$, left trapezius $(p<0.0002)$ and right longissimus $(\mathrm{p}<0.0001)$ when a support is applied to the dentist's abdomen. The left longissimus was the only one to show no change in electrical activity.

In the analysis of adopted positions, the 9 o'clock position indicated a change in the electrical activity of only the right longissimus $(\mathrm{p}<0.05)$ whereas the 10 o'clock position showed a significant difference in both trapezii $(p<0.05)$. Ultimately, the 11 o'clock position only had a change to the left superior trapezius $(\mathrm{p}<0.05)$.

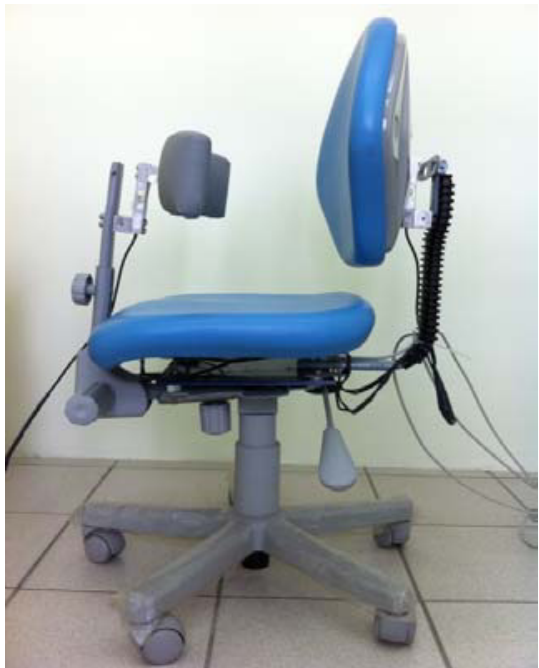

Figure 2: Modified and instrumented dental stool

With regard to the distribution of body weight, there was an increase in the front of the seat rising from an average of $30.8 \pm 2.1 \%$ to $32.83 \pm 2.49 \%$ and a reduction in the back of the seat of the same from $50.20 \pm 2.81 \%$ to $48.6 \pm 4.4 \%$. However, this change was not statistically significant. The backrest also had no significant change in the registered load. The average load registered was $4.3 \pm 4.1 \%$ of body weight for the stool without an abdominal support and $4.5 \pm 4.8 \%$ with the abdominal support. The ab- 
dominal support registered $3.1 \pm 1.9 \%$ of the dentists' body weight.

\section{Discussion}

All the volunteers presented flexion of the cervical spine due to the preference for a direct vision. The electrical activity of the superior trapezius and longissimus thoracic muscles was higher in dentists who used the 10 and 11 o'clock positions. This increase in activity has been observed in direct vision when compared to other alternative methods to view the oral cavity, such as magnifiers and monitors [6]. On the other hand, the 9 o'clock position showed no increase in electrical activity of the trapezii. This confirms that it is the most adequate positions to work in, as it is already more than recommended by the ISO/FDI, since the arms are closer to the trunk.

Regarding the corporal distribution, the weight increase in AA is due to an increased lumbar curve which transfers pressure from the ischial tubersities to the back of the thighs [7].

The AbS was considered by most of the volunteers as a promoter of a more appropriate posture. This same benefit has already been pictured for the lumbar spine considering the use of a support for the trunk [1].

\section{Conclusions}

The use of the AbS in a dental stool during clinical restorative procedures did not significantly interfere in weight distribution in the FS, BS and $\mathrm{Br}$, but did indicate that if a support were applied to the trunk, the support itself would be used and interfere with the electrical activity of most of the muscles studied promoting a position closer to the central axis of the body and therefore protecting the passive elements of the lumbar spine in the sample population of this study.

\section{Acknowledgements}

Financial support: The Coordination for the Improvement of Higher Education Personnel (CAPES).

\section{Reference}

[1] D.J. Ahearn, M.J. Sanders, C. Turcotte, Ergonomic design for dental offices, Work. 35(2010), 495-503.

[2] J.L. Hardage, J.R. Gildersleeve, J.D. Rugh, Journal of the American Dental Association, 107(1983), 937-939.

[3] T. Kihara, Kurume Medical Journal, The Dental Care Works and Work-Related Complaints of Dentists, 42(1995), 251-257.

[4] M.J. Sanders, C.M. Turcotte, Work Occupational stress in dental hygienists, 35(2010), 455-465.

[5] SENIAM, Disponível em < http://www.seniam.org/>

[6] C.A. Smith, C.M. Sommerich, G.A. Mirka, M.C. George, An investigation of ergonomic interventions in dental hygiene work, Applied Ergonomics, 33(2002), 175-184.

[7] D. Zacharkow, Posture: sitting, standing, chair design, and exercise, Charles C. Thomas Publisher, Springfield, 1988. 